\title{
Portrait du Père Noël en Docteur Faust
}

Savoir, pouvoir, innocence et émerveillement dans Santa Claus de E. E. Cummings

\section{Carole Rebillon}

\section{(2) OpenEdition}

1 Journals

Édition électronique

URL : http://journals.openedition.org/aes/322

DOI : 10.4000/aes.322

ISSN : 2258-093X

Éditeur

Laboratoire LISAA

Référence électronique

Carole Rebillon, «Portrait du Père Noël en Docteur Faust », Arts et Savoirs [En ligne], 5 | 2015, mis en ligne le 15 mars 2015, consulté le 01 mai 2019. URL : http://journals.openedition.org/aes/322 ; DOI : $10.4000 /$ aes.322

Ce document a été généré automatiquement le 1 mai 2019.

Centre de recherche LISAA (Littératures SAvoirs et Arts) 


\section{Portrait du Père Noël en Docteur}

\section{Faust}

Savoir, pouvoir, innocence et émerveillement dans Santa Claus de E. E. Cummings

\section{Carole Rebillon}

1 En 1946, le poète américain E. E. Cummings rédige une courte pièce de théâtre, Santa Claus, A Morality, présentée comme une fantaisie de Noël, un divertissement burlesque. Cummings avait déjà tenté plusieurs fois de créer une œuvre dramatique, mais Santa Claus est l'une des deux seules tentatives abouties. Il s'agit au demeurant d'une œuvre théâtrale extrêmement brève, en cinq scènes. Elle comporte peu de personnages: quatre personnages individualisés, Death (« Mort », personnage masculin ${ }^{1}$ ); Santa Claus (le Père Noël) ; Child (l'Enfant) ; Woman (la Femme). Mob (la Foule), composée d'êtres humains non individualisés, peut de ce fait être considérée comme un cinquième personnage.

2 Le sous-titre donné par Cummings à sa pièce, "A morality ", l'apparente explicitement aux morality plays, des spectacles allégoriques très populaires au Moyen-Âge et à la Renaissance. Dans l'une de ses six nonlectures, prononcées à Harvard en 1953, l'auteur désigne Santa Claus par l'expression « ma petite allégorie en vers blancs " ${ }^{2}$, et assume cette parenté ainsi que le message qui s'en dégage. En même temps, il minimise l'importance de cette œuvre et met l'accent sur sa brièveté, comme une excuse ou comme si son écriture avait été un jeu. Santa Claus ne serait-elle qu'un accident au milieu de la profusion de poèmes que comporte le corpus cummingsien? Selon le biographe de E. E. Cummings, R. D. Kennedy, la place de cette œuvre burlesque serait dans un théâtre de marionnettes plutôt que sur une "vraie » scène de théâtre ${ }^{3}$. Or, dès la première scène, dans le dialogue initial entre Mort et le Père Noël, le caractère burlesque s'efface et laisse place à un questionnement plus grave mais présenté sur un ton faussement léger: comment l'imaginaire (incarné par le Père Noël) peut-il survivre et trouver une utilité, dans un monde matérialiste obnubilé par le savoir au détriment de la compréhension ? La parodie du mythe de Faust sur laquelle la pièce est basée nous conduit à porter sur elle un autre regard et à nous interroger sur les enjeux du marché faustien conclu entre Mort et 
le Père Noël, notamment ceux touchant à la représentation du savoir et de la science. De plus, en étudiant la manière dont Cummings fait d'une technologie fictive l'un des ressorts principaux de son intrigue, nous aurons un aperçu de sa réflexion sur la valeur du discours scientifique compte tenu de sa nécessaire adaptation au public à qui il est adressé auquel il s'adresse, ainsi que du rapport du poète au savoir et à la technologie.

\section{Portrait du Père Noël en Docteur Faust}

Ainsi que le note R. S. Kennedy, l'intrigue de Santa Claus, basée sur un marché conclu entre les deux personnages principaux, est une variation aisément reconnaissable du mythe de Faust ${ }^{4}$ : en échange d'un conseil qui lui permettra de résoudre son problème «de distribution", Mort impose, plus qu'il ne propose, au Père Noël d'intervertir les masques puis les costumes dans la scène 3. Il lui offre l'occasion de se sentir autre en devenant pour un moment un "Scientifique » maître de tous les savoirs, dont la maitrise permet de manipuler la Foule. Ce qui apparente le marché entre Mort et le Père Noël à celui passé entre Faust et Mephistopheles dans Doctor Faustus de Marlowe ${ }^{5}$ est son enjeu : une forme de pouvoir basé sur l'acquisition d'un savoir, voire (dans le cas de Doctor Faustus) de la Connaissance ultime et complète des rouages de l'Univers. Ce marché est passé entre un personnage naïf et un initiateur maléfique, qui exige de son élève qu'il lui offre son identité en échange. Cependant, des différences de degré notables interviennent entre les deux marchés faustiens: là où Faust échangeait son âme contre un savoir présenté comme réel bien qu'ésotérique, donc réservé à une élite et procurant à celui qui le possède un pouvoir quasi-illimité sur les êtres et les choses, le Père Noël ne donne qu'un masque en échange de consignes lui permettant de mettre en scène une représentation de la Science sous la forme d'un simple discours, sans manifestations extraordinaires d'aucune sorte. La parodie est visible également si on compare la scène dans laquelle Faust fait apparaître devant l'Empereur les fantômes de personnages antiques $^{6}$ et celle où le Père Noël, après un long discours adressé à la Foule, est censé, d'après les répliques, distribuer des actions de la mine de rouages ${ }^{7}$. En l'absence de didascalie, rien ne dit qu'il donne véritablement quelque chose, ne serait-ce qu'un bout de papier, aux individus de la Foule. Dans Santa Claus, le discours bien mis en scène suffira au Père Noël pour berner une Foule crédule ; au contraire, dans Doctor Faustus l'Empereur et sa suite demandent et obtiennent de l'apprenti sorcier qu'il fasse la preuve de ses dires et de ses pouvoirs de nécromancien. Le discours ne leur suffit pas : ils veulent voir.

Dans Doctor Faustus comme dans Santa Claus, la représentation du savoir est partielle: chez Marlowe, Faust rejette les livres savants, incomplets à ses yeux, et n'en lit que des bribes $^{8}$; plus tard, l'initiation effectuée par Mephistopheles sera elle-même incomplète. Tout le discours de son initiateur consiste en une suite d'affirmations de choses déjà connues du spectateur un peu instruit de l'époque. Ainsi, dans l'acte 2 scène 3, lorsque Faut est enfin autorisé à interroger Mephisopheles, il s'empresse de lui poser des questions sur le fonctionnement de l'univers. Cependant, les réponses qu'il obtient le déçoivent d'autant plus qu'il s'aperçoit qu'il peut les anticiper :

Faust: Mais ont-ils [les astres] tous un mouvement, à la fois situ et tempore?

Mephisopheles: Tous se meuvent d'est en ouest en vingt-quatre heures autour des

pôles du monde, mais diffèrent quant à leurs mouvements autour des pôles du

zodiaque.

Faust: Ces questions simples, Wagner peut les résoudre.

Mephisopheles n'a-t-il aucun talent plus grand que le sien? 
Qui ne connaît le double mouvement des planètes?

Que le premier dure une journée pleine,

Et quant au second : trente jours pour Saturne,

Douze jours pour Jupiter, quatre pour Mars, une année pour le soleil, Vénus et

Mercure, vingt-huit jours pour la lune. Ce sont des questions d'étudiants de

première année. ${ }^{9}$

Les autres secrets ésotériques devant demeurer inconnus, rien ne peut filtrer : c'est de la magie, c'est-à-dire un savoir hermétique, dont les effets ne peuvent être expliqués au grand nombre. Mephistopheles lui-même l'affirme dans la suite de son échange avec Faust :

Faust : [...] Maintenant dis-moi qui a fait le monde.

Mephisopheles : Je ne te dirai pas.

Faust: Scélérat, ne t'ai-je pas lié par serment pour que tu me dises tout?

Mephisopheles: Oui, tout ce qui n'est pas contraire à notre royaume. ${ }^{10}$

6 Rien ne peut donc en être dit sur scène, ce qui exempt l'auteur de plus de précision sur ces savoirs occultes, sans mettre en péril une certaine forme de vraisemblance - du moins, pour le spectateur de l'époque. C'est pourquoi la représentation d'un quelconque savoir réel en tant que tel n'a pas d'intérêt en soi et peut (ou doit) être fragmentaire. Il est permis d'imaginer que, pour les spectateurs de l'époque de la création du Doctor Faustus, le savoir magique maîtrisé par Faust n'est ni inexistant ni inconsistant, ni même fictif : il est simplement irreprésentable, d'autant plus que flotte autour de cette science une odeur de soufre, comme des relents de l'Enfer dans lequel Faust plongera fatalement. ${ }^{11}$

Dans la pièce de Cummings, si ce qui est offert par Mort au Père Noël en fait de « savoir » ("Knowledge") n'est là encore qu'une fiction pure et simple, ce fait est pleinement assumé par les personnages eux-mêmes :

Mort: [...] Mais rappelez-vous bien: moins une chose existe, plus les gens en veulent.

Le Père Noël : C'est malheureux, je n'arrive pas à trouver une chose qui n'existe pas. Pouvez-vous m'aider?

Mort : Pourquoi pas une mine de rouages?

Le Père Noël : Une mine ... de rouages?

Mort: Bien sûr, voyons : une mine de rouages n'existe pas, n'a jamais existé, et n'existera jamais. ${ }^{12}$

8 Cette «science » n'a aucune réalité et ne prétend pas en avoir. Mort peut donc affirmer (ironiquement) que c'est le mot de "science" lui-même, et uniquement lui, qui est magique, non pas le savoir qu'il est censé désigner, puisque ce savoir est inexistant : « [...] dès que les gens entendent le mot magique : "Science" [...] vous leur vendrez n'importe quoi sauf la compréhension. » (p. 21).

Prononcer ce mot produit sur l'auditoire des effets que seuls ceux qui maitrisent le discours peuvent (éventuellement) comprendre ou en tout cas provoquer, à défaut de les contrôler par la suite - ce qui est une caractéristique des actions magiques également dans la pièce de Marlowe. Bien plus, ce mot conférera à lui seul tout pouvoir au discours du Père Noël. Ce n'est donc pas ici le caractère secret du savoir qui permet à l'auteur d'être exempté d'une quelconque précision scientifique, mais la vacuité du discours scientifique en tant que tel, lequel n'est en l'occurrence qu'un moyen de manipulation sciemment utilisé. L'enjeu de la représentation du savoir ne porte pas sur les effets d'un savoir réel quoique mystérieux sur ceux qui le maitrisent ou qui tentent de le faire ${ }^{13}$. Cummings s'intéresse plutôt à l'effet d'un discours prétendument savant mais vide de sens sur un auditoire non averti. La représentation du savoir est partielle, en ce qu'elle ne 
s'intéresse qu'à un aspect, la mise en place et la maitrise du discours "savant », sans aucune considération pour le contenu, qui passe au second plan au point d'être remplacé par l'exposé d'une technologie fictive.

\section{Création de la mine de rouages}

10 La création de la mine de rouages se déroule en trois temps : constatation d'un problème ; analyse des raisons du problème ; proposition d'une solution à mettre en œuvre.

11 D'emblée, Mort fait un premier diagnostic en se plaçant sur un plan purement matériel, voire économique : «Moi aussi, j'ai un problème de distribution, mais c'est le vôtre à rebours [...] C'est que moi, j'ai tant à prendre et personne ne veut donner.» (p. 9). Mort évacue ainsi le côté sentimental exprimé par le Père Noël dans sa deuxième réplique et exprime son cynisme dès cette première analyse.

12 Comme explication du problème posé par le Père Noël, Mort propose un raisonnement par l'absurde, en commençant par décrire un monde inimaginable du point de vue du Père Noël, car totalement contraire à celui de l'enfance qu'il symbolise. Ce monde présenté comme imaginaire est simplement une caricature du monde adulte, celui que ne peut plus imaginer le Père Noël et que ce dernier ne peut même pas concevoir. Les habitants du monde décrit par Mort ressemblent d'ailleurs à s'y méprendre aux dépourvus de conscience que Cummings oppose à lui-même et aux lecteurs qui accueillent son œuvre, dans l'introduction du recueil New Poems, publié en $1935^{14}$. Dans ces pages, les "mostpeople", que l'on pourrait traduire par «laplupartdesgens", incarnent tout ce que Cummings rejette, en particulier le matérialisme et le consumérisme, caractéristiques qu'ils partagent avec la Foule de Santa Claus. Or, la caractéristique du monde adulte qui regroupe toutes celles énumérées par Mort dans sa première tirade, c'est précisément le matérialisme, voire le mercantilisme ; rien ne peut se donner, mais ou parce que - tout se vend. En d'autres termes, rien n'existe que ce qui peut être perçu, saisi (au sens figuré comme au sens propre) par le grand nombre. Ce monde obnubilé par la matérialité, foncièrement égoïste, est selon les mots de Mort « un monstre de négation idiot $»^{15}$, auquel on ne peut faire le don de la Compréhension ("Understanding"). La raison invoquée est que la Compréhension est la seule chose qui ne peut se vendre: il s'agit d'une capacité abstraite, impossible à matérialiser, et qui de plus implique une démarche personnelle. Or, les habitants du non-monde réel décrit par Mort, n'ayant pas d'existence propre en tant qu'individus, en sont incapables. S'ils ne sont intéressés que par l'accumulation de choses "matérielles ", la Compréhension (immatérielle) leur échappe inéluctablement. Ne leur reste que l'accumulation de données, sans le pouvoir de les mettre en relation : c'est ce que Mort désigne par le terme "Knowledge", la Connaissance. Ces données peuvent être matérialisées (quelle que soit la forme prise), puis collectées. La prétendue matérialisation de la Connaissance ne peut donc se faire que sous la forme de quelque chose qui sera facilement imaginable, mais qui n'aura d'existence que pour la Foule. En effet, un non-monde ne peut concevoir que des non-choses, des fantômes, c'està-dire des fantasmes, que la Foule considérera comme presque plus réels qu'elle-même. Ainsi que le déclare Mort à la fin de sa tirade, le monde de la Foule est « un monde si faux, si frivole, si inexistant qu'auprès de lui un fantôme est solide $»^{16}$. L'exclamation du Père Noël à la fin de la scène rejoint cette assertion: «Une mine de rouages? Mais c'est parfaitement fantastique ! $»^{17}$ 
13 Autrement dit: c'est extrêmement ingénieux, car Mort paraît avoir saisi toutes les données du problème pour les réunir dans cette simple solution, et c'est un fantasme au sens premier du terme, un fantôme, une non-matérialisation. Cela peut être rapproché d'une réplique de la pièce de Marlowe, adressée par Faust à l'empereur fasciné par les formes humaines que le savoir ésotérique du mage (en fait, les pouvoirs de Mephistopheles) fait apparaître devant ses yeux : "Ce ne sont que des ombres, sans substance aucune. ${ }^{18}$ Rien de tout cela n'est tangible, rien n'est réel. Il en sera de même pour les actions dans la mine de rouages : à l'instar de Mort, la science entendue comme ensemble de connaissances ne peut créer que des simulacres, à l'usage d'un mondesimulacre. Dans ce cadre, ni Mort ni le Père Noël ne peuvent plus être vraiment ce qu'ils sont : ils n'ont plus qu'à échanger leurs costumes, ce qui se produira à la fin de la scène $4^{19}$ - Le Père Noël n'a pas d'autre choix que de mentir doublement, c'est-à-dire de travestir son discours habituel et trahir les valeurs qu'il représente traditionnellement, au bénéfice d'un discours lui-même mensonger, que Mort lui met pour ainsi dire sur les lèvres en même temps que son masque.

\title{
La mine de rouages et le Père Noël marchand de savoir : un double simulacre
}

14 Si la mine de rouages est une représentation du discours savant ou des productions de la Science, Mort et le Père Noël sont conscients qu'il s'agit d'un simulacre, même si visiblement, le Père Noël l'oublie en cours de route. Dans son premier discours, le Père Noël, porté par un enthousiasme qui n'est peut-être pas feint, ira jusqu'à se réjouir de ce que la Science, grâce à son infaillibilité, produit une nouvelle sorte d'humains voire de surhumains :

\begin{abstract}
Le Père Noël: Au temps où l'homme n'était que l'homme, et rien d'autre, qu'est-ce que c'était que l'égalité ? Un mot. Un rêve. Les hommes ne pouvaient être égaux. Pourquoi ? Parce que l'égalité est l'attribut des surhommes, comme vous, et vous, et vous, et vous. Et donc (surmesdames et surmessieurs) lorsque l'oreille impartiale de la Science entend vos voix surhumaines qui crient: «Faites passer!» la Science, dans Sa toute-puissance, répond: «Qu'il y ait des parts privilégiées pour tout le monde ! $»^{20}$
\end{abstract}

Pour la Foule que le Père Noël rencontre à la scène 2, le certificat de propriété d'une action à tarif spécial est le signe tangible de l'existence de la mine :

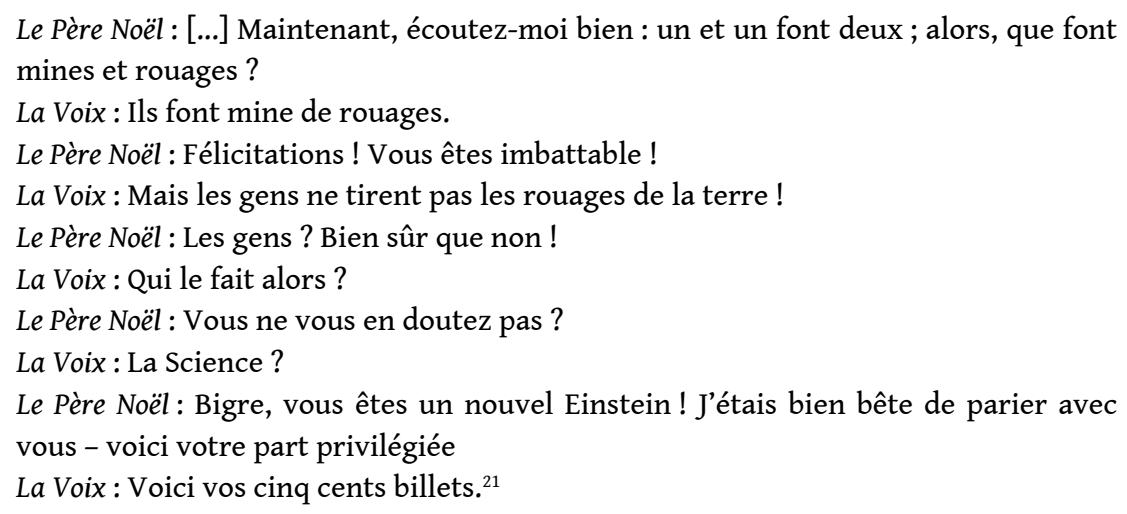

Comme l'écrit Baudrillard, affirmation que peut s'appliquer au texte analysé «Il s'agit d'une substitution au réel des signes du réel [...]. $»^{22}$ Le signe proposé est perceptible, puisque la feuille de papier peut être saisie : l'objet et les signes qu'il est censé porter sont 
une preuve d'existence. Remarquons au passage que nulle didascalie ne précise que le Père Noël a effectivement ces certificats dans la main: le geste peut suffire, l'interprétation du metteur en scène reste libre sur ce point. Un Père Noël les mains vides (image qui contraste avec sa représentation traditionnelle) renforcerait l'effet et mettrait en valeur la naïveté de la Foule, dont le raisonnement peut être ainsi résumé : le Père Noël se présente comme un Scientifique ("Je suis l'homme de Science!», p. 27), et sa parole ne saurait être mise en doute, en vertu d'un préjugé ainsi exprimé, avec beaucoup d'ironie, par Socrate, dans Théétète, ou de la Science: «Il est improbable, en vérité, que radote un savant homme ! $»^{23}$

Puisque le Père Noël, savant homme s'il en fut, accomplit le geste de tendre le certificat, alors ce dernier existe matériellement et ce qui y est inscrit est digne de foi. Ce geste prouve dans le même temps l'existence des actions dans la mine de rouages, celle de la mine de rouages elle-même, et enfin du savoir et de la technologie qui rendent possible l'extraction des rouages. La Foule prend pour preuve d'existence du savoir ce qui n'est qu'un signe sans valeur si sa signification n'est pas vérifiée : c'est l'exact contraire d'un raisonnement scientifique valable. C'est vraiment confondre existence du signe (existence virtuelle, qui plus est, si on peut se permettre un oxymore) et réalité de l'objet signifié. Mais peut-il en être autrement dans un monde qui n'est lui-même qu'un simulacre, habité par des humains qui n'ont d'humain que le nom, semble demander Cummings.

Ce simulacre de connaissance est visiblement une science véritable pour la Foule, suivant en cela l'avis de Théétète, le jeune et naïf interlocuteur de Socrate :

Socrate [s'adressant à Théétète] : Bien plutôt, explique-toi bien et vaillamment : à ton avis, qu'est-ce que la connaissance ? [...]

Théétète: Eh bien! mon avis est que ce sont des connaissances, les choses qu'on apprendra de Théodore: la géométrie aussi bien que les connaissances que tu passais tout à l'heure en revue, et encore, d'un autre côté, que la cordonnerie et les autres arts des gens de métier, tous ensemble aussi bien que chacun d'eux à part, ne sont pas autre chose qu'une connaissance. ${ }^{24}$

19 À quoi Socrate répond en substance que Théétète présente, en lieu et place de la Connaissance, divers savoirs et compétences techniques qui ne peuvent prétendre représenter le savoir dans sa globalité. À ce compte, on peut noter que le Père Noël, lui aussi, présente comme Science totale ce qui n'en serait qu'une partie, un simple savoir technique si la mine de rouages existait. Le simulacre est donc double, et la tromperie aussi. Cependant, pour la Foule, la méprise va encore plus loin : non seulement les actions dans la mine de rouages représentent confusément le savoir technique en tant que tel, voire le savoir, mais à vrai dire, celui-ci n'est qu'une abstraction lointaine, à laquelle le Père Noël prête ses traits quoique son visage soit couvert par un masque de Mort. C'est donc le Père Noël qui est désigné par la Foule sous le vocable de "Science». L'identification entre le personnage et ce qu'il représente se manifeste dès la scène 2 , lorsque la Foule acclame le Père Noël. Cela devient encore plus explicite dans la scène 4 : la Foule, persuadée de la véracité d'une rumeur concernant un accident dans la mine qui fait perdre toute la prétendue valeur des actions distribuées par le Père Noël, vilipende et menace de lynchage celui qu'elle portait aux nues deux scènes auparavant :

Père Noël : Mais qui pensez-vous que je suis?

Une autre [voix] : Penser? Nous ne pensons pas : nous savons! Vous êtes la Science!

Père Noël : La Science?

Une voix : La Science - l'escroc qui nous a vendu des parts d'une mine de rouages!

Une autre voix : La Science - le monstre qui enterre les gens vivants ! [...]

Plusieurs voix : Nous, on dit que vous êtes La Science ! À bas La Science !25 
Lême méprise est observée dans la cinquième et dernière scène de la pièce lorsque la Foule exhibe le cadavre de Mort qu'elle vient de lyncher. En effet, la Foule ayant pris Mort pour le Père Noël du fait de l'échange de costumes entre les deux personnages principaux à la fin de l'avant-dernière scène, c'est Mort qui fait les frais de la colère du groupe. La Foule défile donc sur scène tout en criant «L'Homme de Science est mort ! $»^{26}$. La version originale ("Science is dead.") a beaucoup plus de force, puisqu'on peut la comprendre à la fois comme «L'Homme de Science est mort " (traduction retenue par M. Grossman) et «La Science est morte »: le savoir meurt en même temps que celui qui le représente. Cette identification du personnage à ce qu'il est censé offrir démontre une fois de plus l'infantilisme de la foule, l'absence de réflexion individuelle, donc l'absence totale de compréhension, ce qui corrobore les affirmations de Mort à son sujet dans la première scène. L'exigence de la Foule qui confine au caprice, sa naïveté, ses volte-faces, tout cela contribue à rendre plus perceptible le caractère infantile du comportement de ce groupe humain. Le discours du Père Noël, dicté par Mort, se conforme donc au niveau d'exigence de la Foule sur le plan intellectuel, niveau auquel Mort avait préparé le spectateur au début de la pièce.

Cette naïveté conduit la Foule à la révolte et à la scène de lynchage déjà évoquée (scène 4) : les individus, littéralement écrasés par le simulacre, ne cherchent cependant pas à le détruire, inconscients qu'ils sont du caractère fictif, au fond, de ce qu'ils viennent de vivre. Leur ressentiment ne pouvant apparemment s'exprimer que dans l'instant, ils s'en prennent au premier venu qui ressemble au responsable de leurs malheurs fictifs. Ironiquement, l'erreur de la Foule est salutaire, en ce qu'elle la conduit à supprimer le manipulateur à l'origine du simulacre. Rien ne dit que le simulacre lui-même soit détruit dans la tête des gens : au contraire, c'est leur conviction de l'existence réelle du simulacre qui les a poussés au meurtre. La Science est morte, soit, mais qu'en est-il des mines de rouages? Continuent-elles à tourner dans leurs têtes? Cela n'est plus le problème du dramaturge qui préfère se concentrer sur le Père Noël et son humanité pleine et entière enfin recouvrée.

\section{Une science inhumaine : l'inquiétude d'un poète}

Si la Foule ignorante, naïve et cupide, est facilement manipulable, ce qui met l'accent sur la piètre image que Cummings se fait d'une humanité uniquement obsédée par le progrès technologique et par ses apports mais aucunement préoccupée de sa place ou de son rôle dans un monde qu'elle ne peut comprendre, l'image de la mine de rouages montre à quel point le discours scientifique paraît vide de sens à l'auteur. Aussi, avant de conclure, allons-nous revenir brièvement sur les deux discours adressés par le Père Noël à la Foule, qui dressent deux portraits antagonistes de la Science.

Dans le premier discours (scène 2) la Science infaillible paraît inhumaine, en ce sens qu'étant présentée comme infaillible, elle échappe au fameux proverbe «l'erreur est humaine ", et entraînera dans son sillage l'humanité elle-même. Celle-ci deviendra à son tour, grâce à la Science, inhumaine, ou plutôt surhumaine, car elle s'affranchira des contingences et de l'erreur. Ironiquement, le Père Noël s'adresse en ces termes à une Foule dont Mort avait fait auparavant un portrait peu flatteur: la Foule était déjà inhumaine, mais pas pour les mêmes raisons. C'étaient sa cupidité et son matérialisme qui faisaient d'elle un monstre d'inhumanité. Dans son deuxième discours, à la scène 3 le Père 
Noël a perdu sa naïveté : il a compris que la Science n'était qu'un simulacre. La démonstration est faite par l'absurde puisque le Père Noël doit démontrer sa propre inexistence :

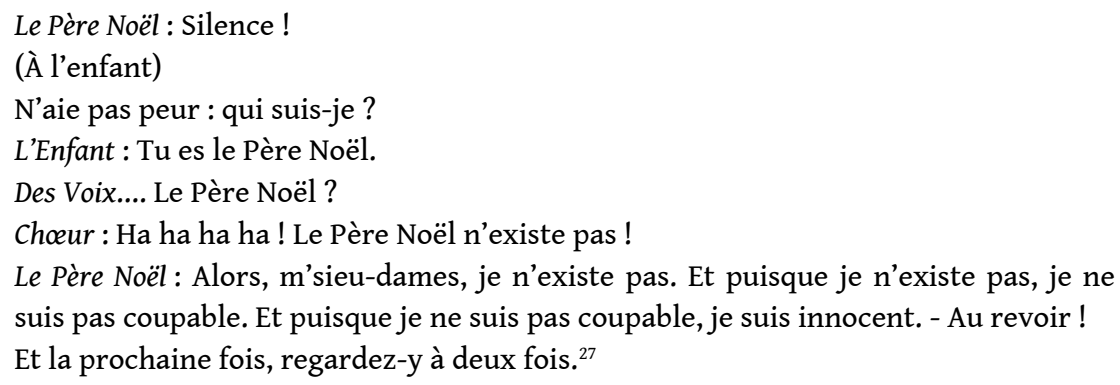

Ce faisant, il montre par conséquent à la Foule que l'erreur existe, alors qu'il avait affirmé dans la scène 2 que la Science permettait à l'humanité d'échapper à ses limites, donc à l'erreur ${ }^{28}$. Cela prouve par contrecoup l'inexistence de la Science comme savoir qu'on ne peut révoquer en doute. Le discours scientifique en ressort vidé de toute substance, devenu presque absurde, lui aussi. Ceux qui l'utilisent sont soupçonnés de ne le faire que dans le but inavoué de manipuler les foules et de gagner à leur cause les véritables êtres humains. Le Père Noël, s'il se rapproche par certains aspects du personnage de Faust, au contraire de son " modèle " réussit à échapper à une forme de damnation en dénonçant son contrat, et il le fait paradoxalement sur les conseils de Mort qui le lui avait mis en main. Ce faisant, il s'éloigne irrémédiablement du monde «inhumain » incarné par la Foule, et il (re)devient véritablement humain. Après cette scène, le Père Noël retrouvera en effet la Femme et l'Enfant qu'il avait perdus, ainsi que la capacité à exprimer ses sentiments véritables. À ce titre, le contraste entre ses discours qui parodient le langage commercial dans les scènes avec la Foule et ses répliques de la quatrième scène lors de son dialogue avec l'Enfant, est très significatif de ce changement. ${ }^{29}$ Non seulement le Père Noël retrouve son vrai visage et les êtres qui lui étaient chers, mais il recouvre la possibilité de ressentir, de reconnaître ses erreurs, de donner, purement et simplement, ce qui était son vœu exprimé au tout début de la pièce.

La désillusion du Père Noël vient à la fois de la prise de conscience du vide de son discours, et de sa dangerosité. Quel crédit accorder à ce discours matérialisé par la mine de rouages, avec toute la vacuité que cette image porte en elle, et qui se présente comme scientifique? L'angoisse du Père Noël fait écho à une inquiétude de l'auteur, à son rejet d'un discours scientifique pour lui vide de sens, et qui ferait de l'humanité un monstre servile. Il faut se rappeler que la pièce est parue en 1946. Cummings, qui n'a pas participé à la seconde guerre mondiale, a exprimé dans certains poèmes son dégoût face à une technologie qui s'est donné pour but la destruction du genre humain, en se mettant entre autres au service de l'armée ${ }^{30}$. Selon lui, dans son introduction au recueil New Poems de 1935, la science rend l'humanité passive, et l'un de ses buts, sinon le seul, est de vendre du progrès sous forme d'objets toujours plus sophistiqués, ce qui, souligne-t-il, empêche les hommes d'accéder à la vraie vie, à la vraie connaissance de ce qu'ils sont :

La vie, pour laplupartdesgens, n'existe simplement pas. Prenez le soidisant niveaudevie. Que veut dire laplupartdesgens par «vie»? Ils ne veulent pas dire « vie ». Ils veulent dire l'approximation collective la plus récente et la plus proche de la passivité prénatale solitaire que la science, dans sa sagesse finie mais sans limites, a réussi à vendre à leurs épouses. ${ }^{31}$

De la même façon, l'un des buts du Père Noël, en faisant sien un discours pseudoscientifique, sera de vendre des choses inutiles à une Foule que son matérialisme prive de 
la Compréhension véritable à laquelle elle ne peut accéder. Cette vision extrêmement méfiante et pessimiste, ce parti pris dont Santa Claus se fait l'écho, sont cependant tempérés par la rédemption du Père Noël et le meurtre du premier émetteur du discours cynique qui présente la science comme un simulacre. À la fin, Mort, incarnant sur scène le côté satirique du Poète, s'efface devant son côté lyrique (le Père Noël) : une connaissance véritable, c'est-à-dire alliée à la compréhension, est peut-être possible, à condition de rejeter toute finalité matérialiste.

Si Cummings critique de façon acerbe le discours scientifique, il se fait peut-être malgré lui l'écho d'interrogations qui sont exprimées, à la même époque, par des scientifiques éminents, sur le but de la science et également sur ce qui peut ou doit être partagé avec le plus grand nombre. C'est ainsi que le physicien Erwin Schrödinger, en 1950, constate et regrette le malentendu entre le grand public et les scientifiques, non seulement sur les buts véritables de la science (et pour ce qui le concernait plus particulièrement, la science physique), mais aussi sur la méprise qui s'ensuit et qui entretient le malentendu :

Beaucoup s'imaginent - dans leur complète ignorance de ce qu'est réellement la science - qu'elle a pour tâche principale la mission auxiliaire d'inventer, ou d'aider à inventer, de nouvelles machines qui amélioreront nos conditions de vie. [...] Si des personnes qui ont cette perspective décident de la formation à donner à nos enfants, le résultat doit être nécessairement celui que je viens de décrire.

Il y a, bien entendu, des raisons historiques qui expliquent pourquoi cette attitude prévaut encore à l'heure actuelle. L'impact de la science sur le fonds idéal de la vie a toujours été très grand, excepté peut-être pendant le Moyen Âge, lorsque la science n'existait pratiquement pas en Europe. Mais il faut reconnaître qu'il y a eu aussi, à une époque plus récente, un phénomène d'obnubilation qui a pu facilement donner le change et faire sous-estimer la tâche idéale de la science. [...] Le fabuleux développement matériel a conduit à une perspective matérialiste, soi-disant appuyée sur les nouvelles découvertes scientifiques. [... ${ }^{32}$

En contrepoint à cette réflexion de Schrödinger sur la nécessité de la vulgarisation et la difficulté découlant des préjugés sur les buts de la science, remarquons que dans la pièce de Cummings, Mort n'envisage de donner à la Foule, en matière de discours pseudoscientifique, que ce qu'elle sait déjà ou ce qu'elle veut entendre. Le portrait de la Foule est à charge: elle est manipulée parce qu'elle le veut bien, et il n'est pas sûr que son ignorance soit une excuse. C'est l'émetteur du discours qui a adapté ce dernier aux exigences de la Foule, et non la Foule qui aurait fait l'effort d'adaptation inverse. Cela conduit à un questionnement qui, peut-être, dépasse celui de l'auteur dans le cadre de cette œuvre, mais que le statut de "morality play" de la pièce amène à se poser : celui du rôle des récepteurs dans l'élaboration du discours scientifique transmis au grand public questionnement abordé par Schrödinger dans le passage cité plus haut. Du fait du caractère allégorique de la pièce, et de l'effort interprétatif qui lui est par conséquent demandé, le spectateur ou le lecteur ne peut que se sentir dans la quasi-obligation de choisir (si possible) entre les trois postures incarnées respectivement par Mort, la Foule et le Père Noël : lucidité qui confine au cynisme, matérialisme, ou émerveillement.

À travers la rédemption du Père Noël, Faust burlesque passant de la naïveté à une lucidité que n'amoindrit pas l'émerveillement, Cummings réaffirme un point de vue déjà exprimé dans de nombreux poèmes : celui du primat de l'imagination, de l'émerveillement et du sentiment sur un savoir uniquement raisonné, qui serait coupé de tout ressenti, de toute intuition. Dans cette pièce ouvertement satirique, les masques et la comédie cachent difficilement l'inquiétude profonde du poète devant des savoirs et des technologies qu'il ne peut maîtriser, symboles pour lui de pouvoir et d'aliénation. 


\section{NOTES}

1. Dans la version originale, Death («Mort»), qui, comme son nom l'indique, personnifie la mort, est un personnage explicitement masculin. Son genre a une importance dans la suite de l'intrigue. Par conséquent, parler de ce personnage au féminin serait ici un contresens : c'est pourquoi nous utiliserons des pronoms personnels masculins pour désigner ce personnage et nous ne mettrons pas d'article devant son nom, comme s'il s'agissait d'un nom propre - tout en ayant conscience d'aller contre l'usage français, qui veut que «mort» soit un nom commun du genre féminin.

2. Dans le texte original: “”. Edward Estlin Cummings, I - six nonlectures, Cambridge, Mass., Harvard University Press, 1953, p. 103. (Ma traduction).

3. Richard S. Kennedy, Dreams In the Mirror: a biography of E. E. Cummings, New York, Londres, Liveright, 1994, p. 407 à 409.

4. Voir Kennedy, op. cit., p. 407: "Santa Claus is really two plays entwined together, one using the plot line of the Faust story, the other the story of lost loved ones reunited." (« Santa Claus est véritablement constitué de deux pièces imbriquées : l'une dont l'intrigue est basée sur l'histoire de Faust, l'autre étant l'histoire d'êtres qui se sont aimés, puis perdus, et qui se trouvent réunis. ») (Ma traduction).

5. Christopher Marlowe, The Tragedy of Doctor Faustus, in Doctor Faustus and Other Plays, Oxford University Press, 1998. J'ai utilisé comme référence le B-Text, daté de 1616 (p. 186-246).

6. Voir Marlowe, op. cit., p. 220 à 224.

7. Voir Cummings, op. cit., p. 26 à 41. La scène ne contient aucune didascalie, hormis celle du tout début précisant que le Père Noël porte le masque de Mort.

8. Voir Marlowe, op. cit., p. 188-189.

9. C. Marlowe, op.cit., p. 204. (Ma traduction). Wagner est le valet au service de Faust.

10. Ibid., p. 205. (Ma traduction).

11. À ce sujet, voir l'ouvrage de Mickaël Popelard, La Figure du savant chez Shakespeare et Marlowe Rêves de puissance et ruine de l'âme, Paris, PUF, 2010, en particulier le chapitre VI intitulé « Faustus ou la science triviale et illusoire ».

12. E. E. Cummings, op. cit., p. 23-25.

13. Voir à ce sujet le prologue de la pièce de Marlowe, op.cit., p. 187.

14. E. E., Cummings, Complete Poems 1904-1962, G. Firmage éd., Liveright, New York, 1991, p. 461-462.

15. E .E., Cummings, Le Père Noël/Santa Claus, op. cit., p. 12-13.

16. Dans le texte original: " $a$ world so false, so trivial, so unso, phantoms are solid by comparison", Ibid., p. 12-14.

17. Ibid., p. 14.

18. "These are but shadows, not substantial". C. Marlowe, op.cit., p. 223. (Ma traduction).

19. E. E. Cummings, Le Père Noël/Santa Claus, op. cit., p. 66-67.

20. Ibid., p. 37 à 41 .

21. Ibid., p. 33-35.

22. Jean Baudrillard, Simulacres et simulation, Paris, Galilée, 1981, p. 11.

23. Platon, CEuvres complètes, vol. 2, Paris, Bibliothèque de la Pléiade, 1950, p. 98.

24. Ibid., p. 89.

25. E. E. Cummings, Le Père Noël/ Santa Claus, op. cit., p. 53.

26. Ibid., p. 81. 
27. Ibid., p. 59-91.

28. Voir Ibid., p. 37-41.

29. Voir Ibid., p. 68-75.

30. C'est le cas en particulier dans le poème "plato told him", qui porte une allusion claire à la politique américaine en matière d'armement à cette période. Voir E. E. Cummings, Complete Poems 1904-1962, op. cit., p. 553.

31. Texte original: "Life, for mostpeople, simply isn't. Take the socalled standardofliving. What do mostpeople mean by "living" ? They don't mean living. They mean the latest and closest plural approximation to singulair prenatal passivity which science,in its finite but unbounded wisdom, has succeeded in selling their wives." Ibid., p. 461. (Ma tradution. J'ai tenté de respecter dans la traduction les particularités orthographiques et grammaticales présentes dans le texte original.)

32. Erwin Schrödinger, Physique quantique et représentation du monde, trad. J. Ladrière, Paris, Seuil, 1992, p. 29-31.

\section{RÉSUMÉS}

En 1946, le poète américain E.E.Cummings fait paraître une brève pièce de théâtre satirique : Santa Claus. A Morality. Cette pièce se présente comme une réécriture burlesque du mythe de Faust, et Cummings en assume le caractère allégorique. Après avoir abordé les rapports entre Faust et Santa Claus, nous étudierons le rôle central que tient l'image d'une technologie imaginaire (la "mine de rouages ») dans l'économie de la pièce. Ce faisant, nous aurons un aperçu d'un questionnement qui parcourt toute l'œuvre poétique de Cummings, celle de la place de l'imaginaire dans un monde matérialiste et envahi par la technologie. Ce questionnement sera mis en relation avec celui de la réception de la vulgarisation scientifique à l'époque de l'écriture de la pièce.

INDEX

Mots-clés : Cummings (Edward Estlin), savoir, technologie

\section{AUTEUR}

\section{CAROLE REBILLON}

